\title{
Young open clusters in the Carina region
}

\author{
Giovanni Carraro, Paola Marigo \\ Dipartimento di Astronomia, Universitá di Padova, \\ Vicolo Osservatorio 2, I-35122 Padova, Italia
}

Paolo Ventura

Osservatorio Astronomico di Roma, Via di Frascati 33, I-00040 Monte Porzio Catone, Italia

Martino Romaniello, and Ferdinando Patat

European Southern Observatory, Karl-Schwarzschild-Straße 2, D-85748 Garching-bei-München, BRD

\begin{abstract}
We present results of a new investigation (Carraro et al. 2002) aimed at clarifying the mutual relationship between the three most prominent young open clusters close to $\eta$ Carinæ, namely Trumpler 16, Trumpler 14 and Collinder 232.
\end{abstract}

\section{Introduction}

We have obtained deep UBVRI CCD-photometry for Trumpler 14, Trumpler 16 and Collinder 232, the three young clusters closest to $\eta$ Carinæ. In this paper we briefly summarize our analysis method and results. First of all we have tried to reconstruct the extinction pattern in the direction of the three clusters. By combining optical photometry with near infrared photometry and spectral classification for a sample of stars in the three clusters, we find using a variety of methods that the the total to selective absorption ratio varies from cluster to cluster, being $R_{V}=3.48 \pm 0.11,4.16 \pm 0.07$ and $3.73 \pm 0.01$ for Trumpler 16 , Trumpler 14 and Collinder 232, respectively. Then, by using the method devised by Romaniello et al. (2002), we derive individual star color excesses and built up reddening corrected color-magnitude diagrams (CMD), from which we constrain clusters's distances, finding that Trumpler 14, Trumpler 16 and Collinder 232 are located $2.9 \pm 0.3,4.4 \pm 0.3$ and $2.5 \pm 0.3 \mathrm{kpc}$ from the Sun, respectively. The method devised by Romaniello et al. (2002) provides also stars luminosities and effective temperatures, that we use to construct the H-R diagram for the clusters, which together with CMDs, have been used to derive clusters' ages and age spreads. To this aim pre-Main Sequence isochrones have been adopted from Ventura et al. (1998). We find that Trumpler 16 is older than Trumpler 14 and that all the clusters have a prominent pre-MS population. As an example, in 

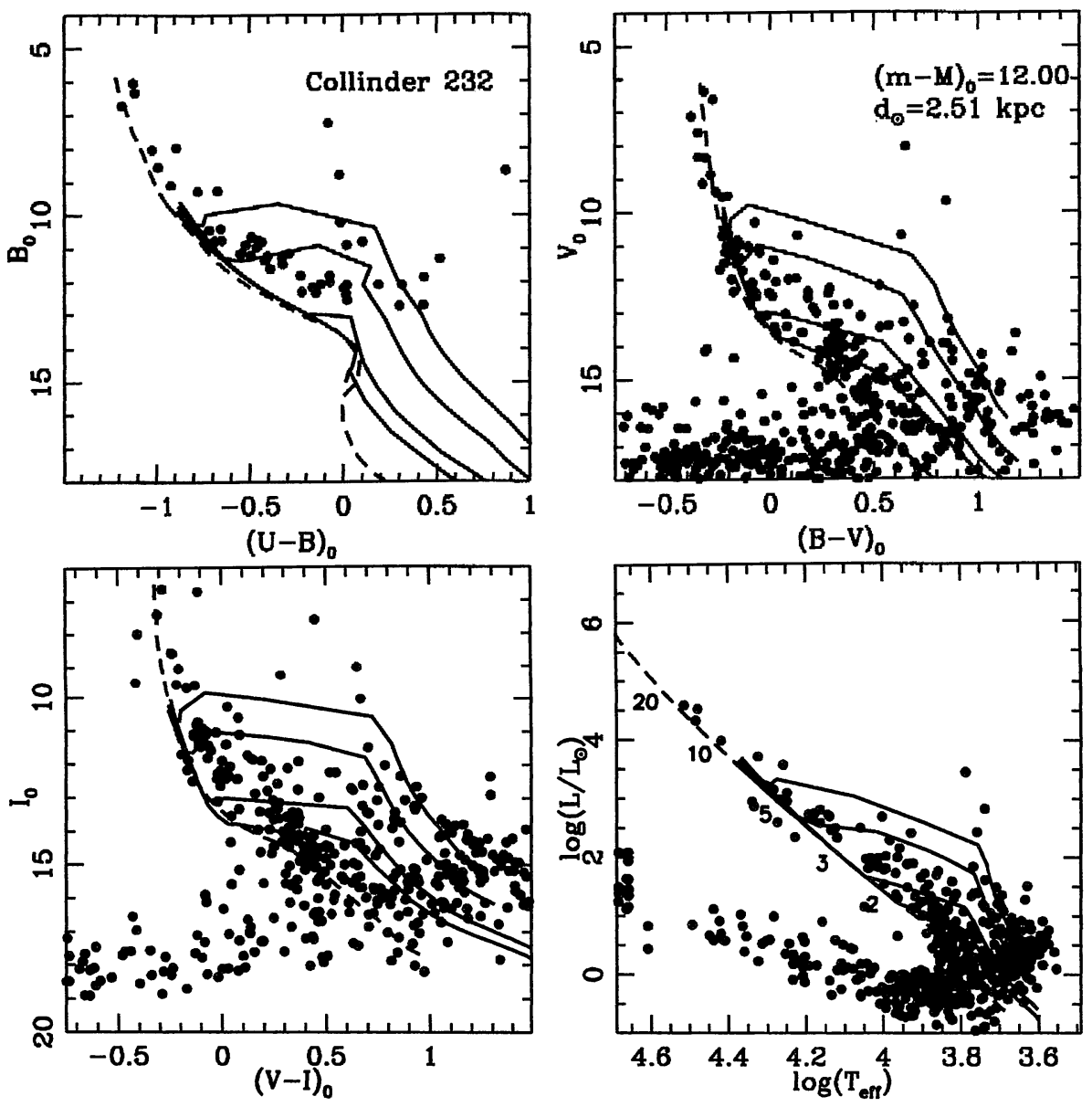

Figure 1. CMDs and H-R diagram for Collinder 232. The dashed line is an empirical ZAMS, the solid lines are pre-MS isochrones $\left(5 \times 10^{5}, 1,5\right.$ and $\left.10 \times 10^{6} \mathrm{yr}\right)$.

Figure 1 we present our results for Collinder 232, that we conclude to be a real open cluster.

\section{References}

Carraro, G., Ventura, P., Romaniello, M., Patat, F. 2002, A\&A submitted Romaniello, M., Panagia, N., Scuderi, S., Kirshner, R.P. 2002, AJ 123, 915

Ventura, P., Zeppieri, A., Mazzitelli, I., D'Antona, F. 1998, A\&A 334, 953 